\title{
Interferon $\alpha$-Inducible Protein 27 Computational Network Construction and Comparison between the Frontal Cortex of HIV Encephalitis (HIVE) and HIVE-Control Patients
}

\author{
Juxiang Huang ${ }^{1, \#}$, Lin Wang ${ }^{1, \#, *}$, Minghu Jiang ${ }^{2, \#}$ and Xiguang Zheng ${ }^{3}$
}

\author{
${ }^{1}$ Biomedical Center, School of Electronics Engineering, Beijing University of Posts and Telecommunications, \\ Beijing, 100876, China; ${ }^{2}$ Lab of Computational Linguistics, School of Humanities and Social Sciences, Tsinghua \\ Univ., Beijing, 100084, China; ${ }^{3}$ School of Electrical Computer and Telecommunications Engineering, University of \\ Wollongong, Wollongong, NSW 2522, Australia
}

\begin{abstract}
Interferon $\alpha$-inducible protein 27 (IFI27) computational network construction and analysis of frontal cortex of HIV encephalitis (HIVE) is very useful to identify novel markers and potential targets for prognosis and therapy. Based on integrated gene regulatory network infer (GRNInfer) method by linear programming and a decomposition procedure with analysis of the significant function cluster using Kappa statistics and fuzzy heuristic clustering from DAVID, we identified and constructed significant molecular IFI27 networks from 12 frontal cortex of HIVE-control patients and 16 HIVE in the same GEO Dataset GDS1726. Our integrative results reflected an IFI27 membrane module only in the upstream of the frontal cortex of HIVE-control patients (BTN3A2, RASGRP3, ROR1 inhibition), and the frontal cortex of HIVE (DGKG, LY96 activation; RASGRP3 inhibition); IFI27 organelle only in the upstream of HIVE-control patients (CREB5, OAS1, PDCD4 activation), and HIVE (PDCD4 activation; ZC3HAV1, ZNF652 inhibition); IFI27 sequence variant only in the upstream of HIVE-control patients (ISG15_2, OAS1, TNFRSF11B activation; BTN3A2, LCAT, RORI inhibition), and HIVE (CFB, DGKG, LCAT, LY96 activation; ISG15_2, TNFRSF11B, ZC3HAVI inhibition).
\end{abstract}

Keywords: IFI27, network construction and analysis, the frontal cortex with HIVE, biocomputation.

\section{INTRODUCTION}

The neurodegenerative process in HIV encephalitis (HIVE) is associated with cognitive impairment with extensive damage to the dendritic and synaptic structure. Several mechanisms might be involved including release of neurotoxins, oxidative stress and decreased activity of neurotrophic factors [1]. The effect of HIV on the brain has been studied by several researchers. The investigations include decreased brain dopamine transporters associated with cognitive deficits in HIV patients with or without cocaine abuse; Magnetic resonance imaging and spectroscopy of the brain in HIV disease; Analysis of the effects of injecting drug use and HIV-1 infection on 18F-FDG PET brain metabolism [24]. IFI27 computational metabolism network construction and analysis of the frontal cortex of HIVE is very useful to identify novel markers and potential targets for prognosis and therapy.

IFI27 is one out of 50 genes identified as high expression in the frontal cortex of HIV encephalitis (HIVE) $v s$ HIVEcontrol patients. IFI27's molecular function network contains G-protein coupled receptor, interferon receptor, growth factor, centromere DNA-binding protein, protein phosphatase,

\footnotetext{
*Address correspondence to this author at the Biomedical Center, School of Electronics Engineering, Beijing University of Posts and Telecommunications, Beijing, 100876, China; Tel: 8610-82059506; Fax: 8610-62785736; E-mail: wanglin98@ @tsinghua.org.cn

"Equal contribution
}

phospholipase, metalloprotease, non-receptor tyrosine protein kinase, serine protease inhibitor, non-motor actin binding protein and ubiquitin-protein ligase, and it is relevant to biological process of fatty acid metabolism, mRNA transcription regulation, protein modification, protein phosphorylation, proteolysis, signal transduction, ion transport, immunity and defense and phospholipid metabolism (DAVID database). IFI27's relational study has also been reported previously [5]. However, the molecular mechanism concerning IFI27 network construction in the frontal cortex with HIVE has not been addressed adequately.

This paper is based on our previous publication [6]. Mining larger data sets to get an insight into biological processes at system-wide level has become a challenge for bioinformatics with microarray technologies producing a great deal of gene expression data in the postgenomic era. On the one hand, due to the complexity and distributive nature of biological research, there are several methods for inferring gene regulatory networks but all these methods focus on constructing an entire network calculated from the given microarray data. The large number of genes in those networks makes it is hard to get any clear perception of valuable knowledge from such complicated networks, let alone further study single genes. On the other hand, the wide spread of knowledge from independent databases lowers the study effectiveness. Thus, a novel method of integrating both single molecular network construction and highly centralized gene-functional-annotation analysis is needed for gene network and functional analysis. This paper propose an inte- 
grated method, based on linear programming and a decomposition procedure with integrated analysis of the significant function cluster using kappa statistics and fuzzy heuristic clustering. Our method concentrates on and constructs a prioritized single gene knowledge network integrated with DAVID for the prioritised $(1,2)$ We identified IFI27 activation and inhibition networks, (3) upstream and downstream feedback networks, and (4) IFI27 functional module construction. Our construction of IFI27 network may be useful to identify novel markers and potential targets for prognosis and therapy of HIVE.

\section{MATERIALS AND METHODS}

\section{Microarray Data}

We used microarrays containing 12558 genes from 12 frontal cortex of HIVE-control patients and 16 HIVE in the same GEO Dataset GDS1726 [1]. HIVE-control patients mean normal adjacent frontal cortex tissues of HIV encephalitis (HIVE) and no extensive damage to the dendritic and synaptic structure.

\section{Gene Selection Algorithms}

50 molecular markers of the frontal cortex of HIVE were identified using significant analysis of microarrays (SAM). SAM is a statistical technique for finding significant genes in a set of microarray experiments. The input to SAM is gene expression measurements from a set of microarray experiments, as well as a response variable from each experiment. The response variable may be a grouping like untreated, treated and so on. SAM computes a statistic $d_{i}$ for each gene $i$, measuring the strength of the relationship between gene expression and the response variable. It uses repeated permutations of the data to determine if the expression of any genes is significantly related to the response. The cutoff for significance is determined by a tuning parameter delta, chosen by the user based on the false positive rate. We normalized data by $\log 2$, and selected two class unpaired and minimum fold change $=1.52$. Here we chose the 50 top-fold significant (high expression genes of HIVE compared with HIVEcontrol patients) genes under the false-discovery rate and qvalue as $9.12 \%$. The q-value (invented by John Storey [7]) for each gene is the lowest false discovery rate at which that gene is called significant. It is like the well-known p-value, but adapted to multiple-testing situations.

\section{Network Establishment of Candidate Genes}

The entire network was constructed using GRNInfer [8] and GVedit tools. GRNInfer is a novel mathematic method called GNR (Gene Network Reconstruction tool) based on linear programming and a decomposition procedure for inferring gene networks. The method theoretically ensures the derivation of the most consistent network structure with respect to all of the datasets, thereby not only significantly alleviating the problem of data scarcity but also remarkably improving the reconstruction reliability. The following Equation (1) represents all of the possible networks for the same dataset.

$$
J=\left(X^{\prime}-A\right) U \Lambda^{-1} V^{T}+Y V^{T}=\hat{J}+Y V^{T}
$$

We established network based on the 50 top-fold distinguished genes and selected parameters as lambda 0.0 be- cause we used one dataset, threshold 0.000001. Lambda is a positive parameter, which balances the matching and sparsity terms in the objective function. Using different thresholds, we can predict various networks with different edge density.

\section{Functional Annotation Clustering}

The DAVID Gene Functional Clustering Tool provides typical batch annotation and gene-GO term enrichment analysis for highly throughput genes by classifying them into gene groups based on their annotation term co-occurrence [9, 10]. The grouping algorithm is based on the hypothesis that similar annotations should have similar gene members. The functional annotation clustering integrates the same techniques of Kappa statistics to measure the degree of the common genes between two annotations, and fuzzy heuristic clustering to classify the groups of similar annotations according to kappa values.

\section{RESULTS}

\section{Identification of HIVE Molecular Markers}

IFI27 is one out of 50 genes identified as high expression in frontal cortex of HIV encephalitis (HIVE) vs HIVEcontrol patients. We normalized data by $\log 2$, and selected minimum fold change $=1.5976$. Here we chose the 50 topfold significant (high expression genes of HIVE compared with HIVE-control patients) genes under the false-discovery rate and q-value are $9.12 \%$. We identified potential HIVE molecular markers and chose the 50 top-fold significant positive genes from 12558 genes from 12 frontal cortex of HIVE-control patients and 16 HIVE in the same GEO Dataset GDS1726 including interferon alpha-inducible protein 27 (IFI27), complement factor b (CFB), lymphocyte antigen 96 ( $L Y 96)$, programmed cell death 4 (PDCD4), lecithin-cholesterol acyltransferase $(L C A T)$, ras guanyl releasing protein 3 (RASGRP3), zinc finger ccch-type antiviral 1 (ZC3HAV1), tumor necrosis factor receptor superfamily member 11b (TNFRSF11B), zinc finger protein 652 (ZNF652), diacylglycerol kinase gamma $(D G K G)$, etc. (see List of Abbreviations ).

\section{IFI27 Up- and Down-stream Network Construction in the Frontal Cortex of HIVE-Control Patients and HIVE}

In the frontal cortex of HIVE-control patients, IFI27 upstream network appeared that $A D H 1 B, A F 075680$, CREB5, IFI44L, ISG15_2, OAS1, PDCD4, TNFRSF11B activate IFI27, and BTN3A2, LCAT, RASGRP3, ROR1 inhibit IFI27, as shown in Fig. (1A), whereas in the frontal cortex of HIVE, IFI27 upstream network showed that AL080060, CFB, DGKG, LCAT, LY96, M33210, PDCD4 activate IFI27, and $A D H 1 B, \quad A F 075680$, ISG15_2, RASGRP3, TNFRSF11B, ZC3HAV1, ZNF652 inhibit IFI27, as shown in Fig. (1B). In the frontal cortex of HIVE-control patients and in the frontal cortex of HIVE, IFI27 downstream network showed no results.

\section{Identification of IFI27 Up- and Down-Stream Modules in the Frontal Cortex of HIVE-Control Patients and HIVE by DAVID}

In the frontal cortex of HIVE-control patients, IFI27 upstream modules mainly include membrane (BTN3A2, RASGRP3, ROR1, IFI27), organelle (CREB5, OAS1, PDCD4, 


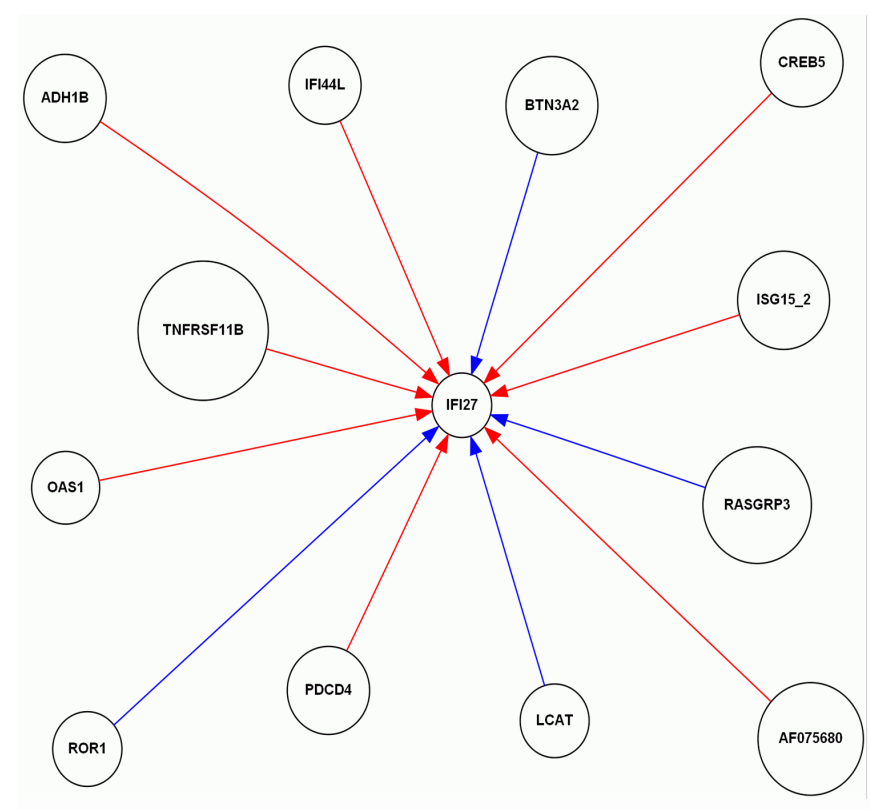

(A)

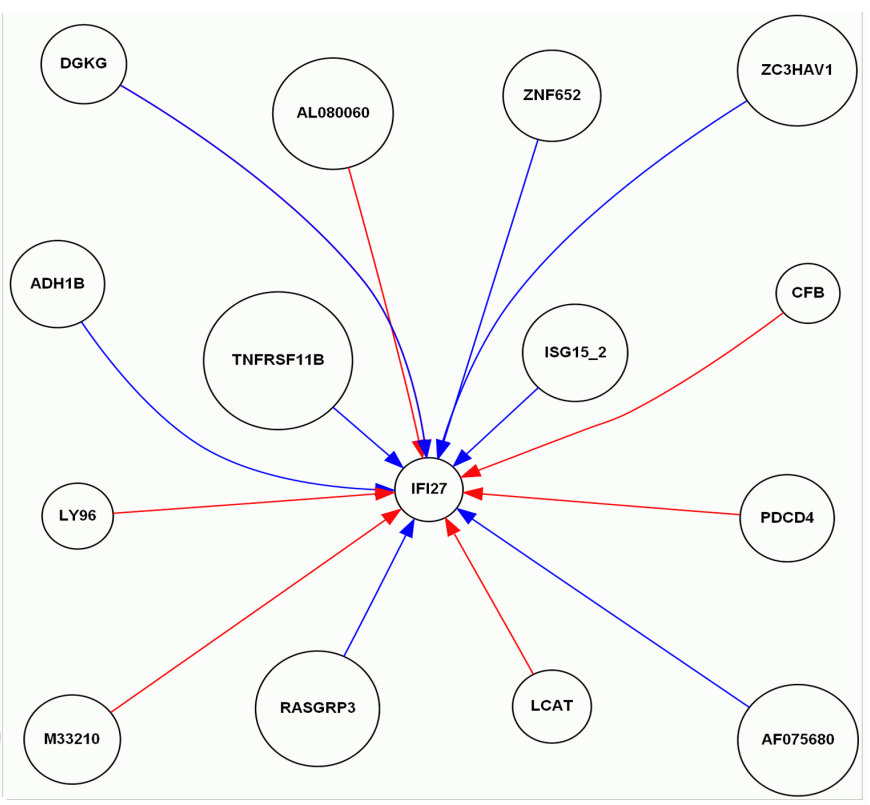

(B)

Fig. (1). IFI27 upstream network construction in the frontal cortex of HIVE-control patients by infer (A). IFI27 upstream network construction in HIVE by infer (B). Red color represents activation, blue color represents inhibition.

IFI27), sequence variant (ISG15_2, OAS1, TNFRSF11B, BTN3A2, LCAT, ROR1, IFI27), etc., as shown in Fig. (2A). In the frontal cortex of HIVE, IFI27 upstream modules mainly cover membrane (DGKG, LY96, RASGRP3, IFI27), organelle (PDCD4, ZC3HAV1, ZNF652, IFI27), sequence variant (CFB, DGKG, LCAT, LY96, ISG15_2, TNFRSF11B, ZC3HAVI, IFI27), etc., as shown in Fig. (2B). In the frontal cortex of HIVE-control patients and in the frontal cortex of HIVE, IFI27 downstream showed no results.

\section{DISCUSSION}

We have previously published work in this field about gene network construction and analysis [6, 11-15] By integration of gene regulatory network infer (GRNInfer) and the database for annotation, visualization and integrated discovery (DAVID 2010 version) we constructed a significant molecular IFI27 network and compared IFI27 up- and downstream gene numbers for activation and inhibition between HIVE-control patients and HIVE (Table 1). Specifically, IFI27 downstream networks showed no results in the frontal cortex of HIVE-control patients and the frontal cortex of HIVE.

In the IFI27 membrane module of the upstream network of frontal cortex of HIVE-control patients, our integrative results suggested that $B T N 3 A 2, R A S G R P 3, R O R 1$ inhibit IFI27, whereas in that of HIVE, DGKG, LY96 activate $I F I 27$, and RASGRP3 inhibits IFI27. For the IFI27 membrane module of the downstream network there was no result (Fig. 1, 2, Table 2). LY96 is identified by molecular function of CAM family adhesion molecule and transmembrane receptor regulatory/adaptor protein, and it is involved in the biological process of mRNA splicing, protein biosynthesis, endocytosis and immunity and defense (DAVID database). LY96's relational study has been reported previously [16-20].
$D G K G$ has been reported to be relevant to molecular function of mRNA processing factor, mRNA splicing factor, kinase, nucleotide kinase, epimerase/racemase, complement component, zinc finger transcription factor, KRAB box transcription factor and RNA helicase, and it is involved in biological process of lipid, fatty acid and steroid metabolism, Acyl-CoA, cholesterol, purine metabolism, mRNA transcription regulation, pre-mRNA processing, mRNA splicing, MAPKKK cascade, T-cell and MHCI-mediated immunity, complement-mediated immunity and other neuronal activity (DAVID database). $D G K G$ 's relational study has also been reported [21-24]. RASGRP3's molecular function consists of select regulatory molecule, G-protein modulator and guanylnucleotide exchange factor, and it is concerned with biological process of mRNA transcription regulation, proteolysis, signal transduction, cell adhesion-mediated signaling, ligandmediated signaling, cell communication and steroid metabolism (DAVID database). RASGRP3's relational study has also bee reported [25-29].

In IFI27 organelle module of upstream network of frontal cortex of HIVE-control patients, our integrative result suggested that $C R E B 5, O A S 1, P D C D 4$ activate $I F I 27$, whereas in that of HIVE, PDCD4 activates IFI27, and ZC3HAV1, ZNF652 inhibits IFI27. In the IFI27 organelle module of downstream network there was no result (Fig. 1, 2, Table 2). PDCD4's molecular function consists of mitochondrial carrier protein, G-protein, large G-protein, guanyl-nucleotide exchange factor, protein kinase, lipase, non-receptor serine/threonine protein kinase, calmodulin related protein, actin and actin related protein, and it is concerned with the biological processes of amino acid catabolism, mRNA transcription, mRNA transcription elongation, mRNA transcription regulation, mRNA splicing, protein metabolism and modification, protein biosynthesis, proteolysis, cell surface receptor medi- 


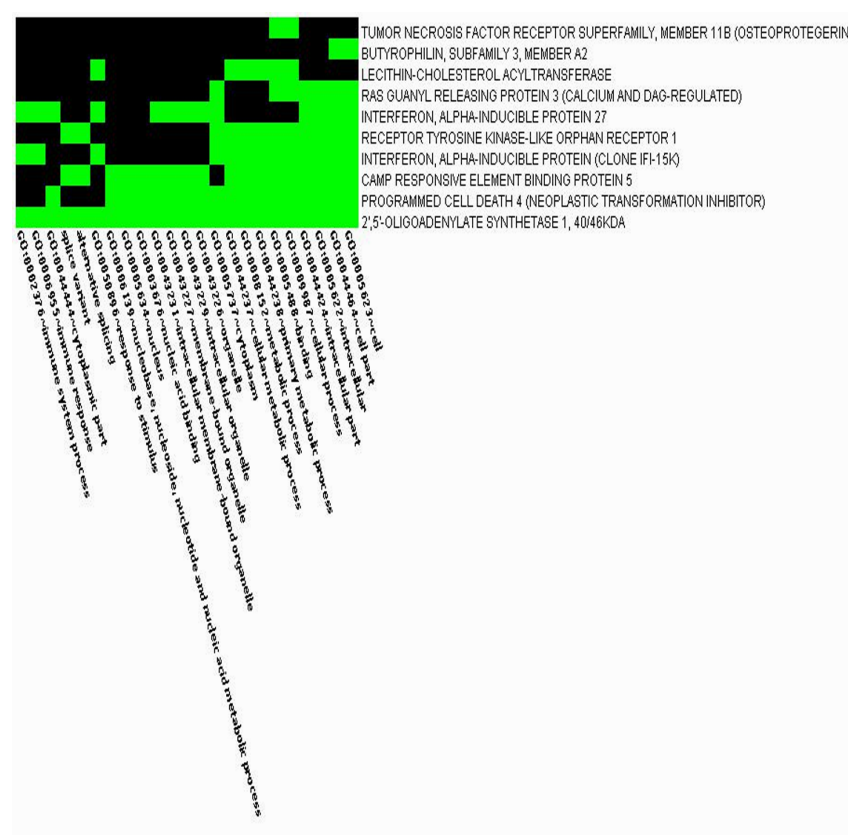

(A)

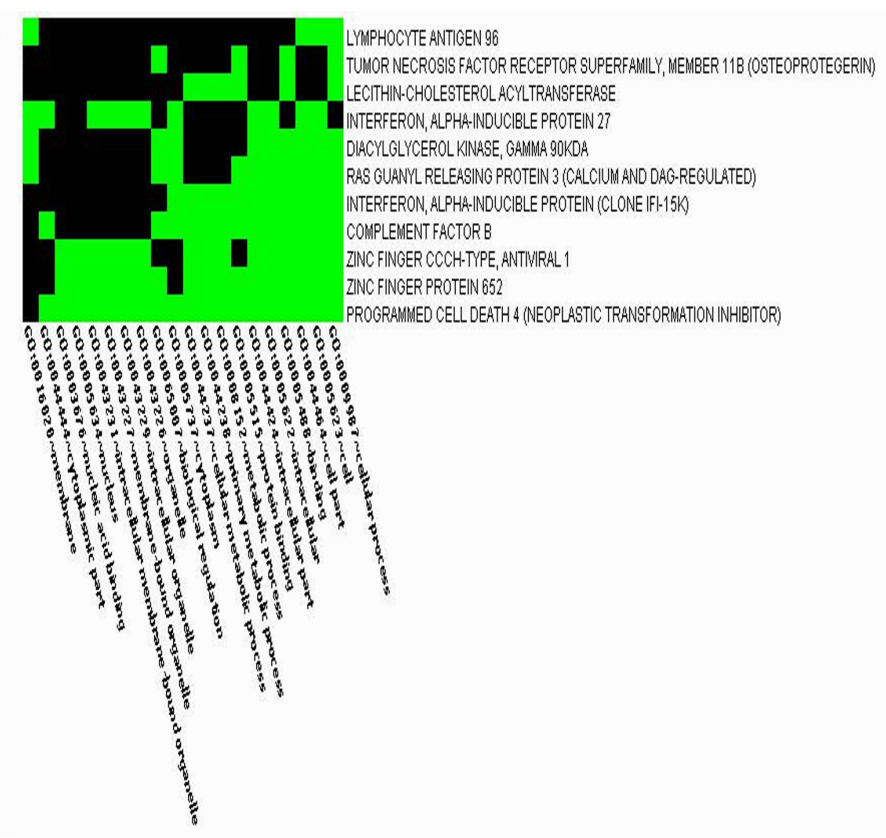

(B)

Fig. (2). IFI27 upstream function modules by DAVID in the frontal cortex of HIVE-control patients (A). IFI27 upstream function modules by DAVID in HIVE (B). Green color represents gene-term association positively reported, black color represents gene-term association not reported yet.

Table 1. IFI27 up- and Down-Stream Gene Numbers of Activation and Inhibition in Each Module Between HIVE-Control Patients and HIVE. Con Represents Control (HIVE-Control Patients), Exp: Experiment (HIVE), Act: Activation, Inh: Inhibition

\begin{tabular}{|c|c|c|c|c|c|c|c|c|}
\hline \multirow{2}{*}{ Term } & \multicolumn{4}{|c|}{ IFI27 Upstream } & \multicolumn{4}{|c|}{ IFI27 Downstream } \\
\hline & $\operatorname{con}(\mathbf{a c t})$ & $\operatorname{con}($ inh $)$ & $\exp (\mathbf{a c t})$ & $\exp (i n h)$ & $\operatorname{con}(\operatorname{act})$ & $\operatorname{con}($ inh $)$ & $\exp (\operatorname{act})$ & $\exp (\mathrm{inh})$ \\
\hline Transmembrane & 0 & 2 & & & & & & \\
\hline Transmembrane Region & 0 & 2 & & & & & & \\
\hline Integral To Membrane & 0 & 3 & & & & & & \\
\hline Intrinsic To Membrane & 0 & 3 & & & & & & \\
\hline Membrane Part & 0 & 3 & & & & & & \\
\hline Intracellular Membrane-Bound Organelle & 3 & 0 & 1 & 2 & & & & \\
\hline Membrane-Bound Organelle & 3 & 0 & 1 & 2 & & & & \\
\hline Membrane & 0 & 3 & 2 & 1 & & & & \\
\hline Intracellular Organelle & 3 & 0 & 1 & 2 & & & & \\
\hline Organelle & 3 & 0 & 1 & 2 & & & & \\
\hline Sequence Variant & 3 & 3 & 4 & 3 & & & & \\
\hline
\end{tabular}

ated signal transduction, G-protein mediated signaling, calcium mediated signaling, small molecule transport, cell proliferation and differentiation, muscle development, chromatin packaging and remodeling (DAVID database). PDCD4's relational study has been reported previously [30-35]. ZC3HAV1 has been reported to be relevant to the molecular function of nuclease, mRNA splicing factor, carbohydrate kinase and endoribonuclease, and it is involved in the biological process of nucleoside catalytic and mRNA transcription regulation (DAVID database). ZC3HAVI's relational study also can be presented in these papers [36-40]. ZNF652 is relevant to molecular function of voltage-gated potassium channel, transcription factor, zinc finger transcription factor, KRAB box transcription factor and non-motor microtubule binding protein, and the biological process of nucleoside metabolism, mRNA transcription, mRNA splicing, protein acetylation and spermatogenesis and motility (DAVID database). ZNF652's relational study has been reported [41-43]. 
Table 2. Activation and Inhibition Gene Names of IFI27 up- and Down-Stream Modules in HIVE-Control Patients and HIVE. Con Represents Control (HIVE-Control Patients), Exp: Experiment (HIVE), Act: Activation, Inh: Inhibition

\begin{tabular}{|c|c|c|c|c|}
\hline Term & $\operatorname{con}(a c t)$ & $\operatorname{con}($ inh $)$ & $\exp (a c t)$ & $\exp (i n h)$ \\
\hline Membrane & & BTN3A2, RASGRP3, ROR1 & $D G K G, L Y 96$ & RASGRP3 \\
\hline Organelle & CREB5, OAS1, PDCD4 & & PDCD4 & ZC3HAV1, ZNF652 \\
\hline \multirow{2}{*}{ Term } & \multicolumn{4}{|c|}{ IFI27 Downstream } \\
\hline & $\operatorname{con}(\mathbf{a c t})$ & $\operatorname{con}($ inh $)$ & $\exp ($ act $)$ & $\exp (i n h)$ \\
\hline \multicolumn{5}{|l|}{ Membrane } \\
\hline
\end{tabular}

In the IFI27 sequence variant module of the upstream network of frontal cortex of HIVE-control patients, our integrative result showed that ISG15_2, OASI, TNFRSF11B activate IFI27, and BTN3A2, LCAT, RORI inhibit IFI27, whereas in that of HIVE, CFB, DGKG, LCAT, LY96 activate IFI27, and ISG15_2, TNFRSF11B, ZC3HAVI inhibit IFI27. In the IFI27 sequence variant module of downstream network there was no result (Fig. 1, 2, Table 2). LCAT is identified by molecular function of interleukin receptor, transferase, acyltransferase, complement component, antibacterial response protein, extracellular matrix structural protein and microtubule binding motor protein, and it is involved in the biological processes of lipid, fatty acid, cholesterol catalytic and synaptic transmission (DAVID database). LCAT's relational study has been reported [44-48]. TNFRSF11B has been shown to be concerned with molecular function of receptors including calcium binding protein and tumor necrosis factor receptor, and skeletal and mesoderm development (DAVID database). TNFRSF11B's relational study has been reported [49-54]. ISG15 is relevant to molecular function of ribosomal protein and annexin, and mRNA transcription regulation, mRNA polyadenylation, protein catalytic and modification, proteolysis and cation transport (DAVID database). ISG15's relational study has been reported [5559]. CFB's molecular function contains protein kinase, methyltransferase, glycosyltransferase, deacetylase, protease, phosphorylase, non-receptor serine/threonine protein kinase, non-receptor tyrosine protein kinase, zinc finger transcription factor, KRAB box transcription factor and non-motor actin binding protein, and it is relevant to biological processes of purine metabolism, mRNA transcription regulation, protein metabolism and modification, protein biosynthesis, protein modification and glycosylation, proteolysis, electron transport, G-protein mediated signaling and cell structure (DAVID database). CFB's relational study has been reported [60-64].
In conclusion, we first identified the significant molecule IFI27 by SAM, then constructed IFI27 up- and down-stream networks by GRNInfer and further data-mined the main IFI27 modules including membrane, organelle and sequence variant from 12 frontal cortex of no-encephalitis HIV patients and 16 HIV encephalitis (HIVE) and in the same GEO Dataset GDS1726 by using DAVID. Our computation showed the different gene rate of IFI27 network in HIVE as $86 \%(12 / 14)$ compared with no-encephalitis HIV patients considering activation and inhibition relationship. Our integrative results reflected IFI27 membrane module only in the upstream of the frontal cortex of no-encephalitis HIV patients (BTN3A2, RASGRP3, ROR1 inhibition), whereas only in the upstream of the frontal cortex of HIVE (DGKG, LY96 activation; RASGRP3 inhibition); IFI27 organelle only in the upstream of no-encephalitis HIV patients (CREB5, OAS1, PDCD4 activation), whereas only in the upstream of HIVE (PDCD4 activation; ZC3HAV1, ZNF652 inhibition); IFI27 sequence variant only in the upstream of no-encephalitis HIV patients (ISG15_2, OAS1, TNFRSF11B activation; BTN3A2, LCAT, ROR 1 inhibition), whereas only in the upstream of HIVE (CFB, DGKG, LCAT, LY96 activation; ISG15_2, TNFRSF11B, ZC3HAVI inhibition) (Table 2).

\section{ACKNOWLEDGEMENTS}

This work was supported by the National Natural Science Foundation in China (No.60871100) and the Teaching and Scientific Research Foundation for the Returned Overseas Chinese Scholars, State Education Ministry. State Key Lab of Pattern Recognition Open Foundation, Major science and technology projects of new transgenic biological breeds (2009ZX08012-001B), Key project of philosophical and social science of MOE (07JZD0005). 
ABBREVIATIONS

\begin{tabular}{|c|c|c|c|}
\hline Gene Symbol & Gene Name & Fold Change & q-value $(\%)$ \\
\hline TNFRSF11B & tumor necrosis factor receptor superfamily member $11 \mathrm{~b}$ & 4.480350846 & 0 \\
\hline OAS1 & 2',5'-oligoadenylate synthetase 1 & 3.935550921 & 0 \\
\hline BTN3A2 & butyrophilin subfamily 3 member A2 & 3.930155645 & 0 \\
\hline IFI44L & interferon-induced protein 44 -like & 3.544133608 & 5.388090171 \\
\hline$A D H 1 B$ & alcohol dehydrogenase 1B (class I) beta polypeptide & 3.267086669 & 0 \\
\hline RASGRP3 & RAS guanyl releasing protein 3 & 3.050957784 & 6.871767174 \\
\hline$A F 075680$ & & 2.980689875 & 9.118306443 \\
\hline ISG15 & ISG15 ubiquitin-like modifier & 2.661333289 & 0 \\
\hline ISG15 & ISG15 ubiquitin-like modifier & 2.634537401 & 9.031465429 \\
\hline МАРКАРКЗ & mitogen-activated protein kinase-activated protein kinase 3 & 2.568139499 & 6.871767174 \\
\hline CREB5 & cAMP responsive element binding protein 5 & 2.430770391 & 0 \\
\hline$M X 1$ & myxovirus resistance 1 interferon-inducible protein $\mathrm{p} 78$ & 2.303681042 & 9.031465429 \\
\hline IFITMI & interferon induced transmembrane protein 1 & 2.288819172 & 4.390295695 \\
\hline MYBPC1 & myosin binding protein $\mathrm{C}$ slow type & 2.269429388 & 0 \\
\hline RORI & receptor tyrosine kinase-like orphan receptor 1 & 2.257714328 & 4.390295695 \\
\hline IFI35 & interferon-induced protein 35 & 2.22216304 & 0 \\
\hline LSM7 & LSM7 homolog U6 small nuclear RNA associated & 2.213979171 & 0 \\
\hline$L C A T$ & lecithin-cholesterol acyltransferase & 2.15393395 & 9.118306443 \\
\hline ZC3HAVI & zinc finger $\mathrm{CCCH}$-type antiviral 1 & 2.110932263 & 9.031465429 \\
\hline$L Y 96$ & lymphocyte antigen 96 & 2.109884854 & 0 \\
\hline TSPAN4 & tetraspanin 4 & 2.097804823 & 9.031465429 \\
\hline C10orf116 & chromosome 10 open reading frame 116 & 2.092862317 & 4.390295695 \\
\hline$D G K G$ & diacylglycerol kinase gamma & 2.087598229 & 9.118306443 \\
\hline STAT1 & signal transducer and activator of transcription 1 & 2.075117894 & 5.388090171 \\
\hline IFI27 & interferon alpha-inducible protein 27 & 2.055270833 & 0 \\
\hline BST2 & bone marrow stromal cell antigen 2 & 2.039254747 & 9.118306443 \\
\hline TGFBR3 & transforming growth factor, beta receptor III & 2.01601052 & 0 \\
\hline SLC16A4 & solute carrier family 16 member 4 & 1.998152827 & 9.031465429 \\
\hline FERIL3 & myoferlin & 1.973894813 & 0 \\
\hline ZNF652 & zinc finger protein 652 & 1.954612806 & 9.031465429 \\
\hline$H L A-B$ & hypothetical protein LOC441528 & 1.937237197 & 9.118306443 \\
\hline PDCD4 & programmed cell death 4 & 1.934462054 & 9.118306443 \\
\hline$S F 1$ & splicing factor 1 & 1.933192211 & 0 \\
\hline$A L 080060$ & & 1.918989403 & 6.871767174 \\
\hline CFHRI & complement factor H-related 1 & 1.83226837 & 9.118306443 \\
\hline$C F B$ & complement factor B & 1.822609627 & 9.118306443 \\
\hline$L G A L S 3 B P$ & lectin galactoside-binding soluble 3 binding protein & 1.810007562 & 9.031465429 \\
\hline
\end{tabular}




$\begin{array}{llll}\text { Gene Symbol } & \text { Gene Name } & \text { Fold Change } & \text { q-value(\%) } \\ \text { CD99 } & \text { CD99 molecule } & 1.794488261 & 9.118306443 \\ \boldsymbol{R D X} & \text { radixin } & 1.763277309 & 9.118306443 \\ \boldsymbol{M T 1 G} & \text { metallothionein 1G } & 1.746025902 & 5.388090171 \\ \boldsymbol{R B B P 6} & \text { retinoblastoma binding protein 6 } & 1.734167688 & 9.031465429 \\ \boldsymbol{T E N C 1} & \text { tensin like C1 domain containing phosphatase } & 1.70355513 & 9.118306443 \\ \boldsymbol{P A X 6} & \text { paired box 6 } & 1.682567328 & 4.390295695 \\ \boldsymbol{N F A T 5} & \text { nuclear factor of activated T-cells 5 tonicity-responsive } & 1.649856939 & 5.388090171 \\ \text { DGKG } & \text { diacylglycerol kinase, gamma } & 1.645823782 & 9.031465429 \\ \boldsymbol{C F D P 1} & \text { craniofacial development protein 1 } & 1.628945094 & 9.031465429 \\ \boldsymbol{V E Z F 1} & \text { vascular endothelial zinc finger 1 } & 1.611704624 & 9.118306443 \\ \boldsymbol{G A S 1} & \text { growth arrest-specific 1 } & 1.597621591 & 9.118306443 \\ \boldsymbol{M 3 3 2 1 0} & & 1.544749215 & 9.031465429 \\ \boldsymbol{A T P 6 V 0 E 1} & \text { ATPase H+ transporting lysosomal 9kDa V0 subunit e1 } & 1.523917145 & 9.118306443\end{array}$

\section{REFERENCES}

[1] Masliah E, Roberts ES, Langford D, et al. Patterns of gene dysregulation in the frontal cortex of patients with HIV encephalitis. J Neuroimmunol 2004; 157: 163-75.

[2] Chang L, Wang GJ, Volkow ND, et al. Decreased brain dopamine transporters are related to cognitive deficits in HIV patients with or without cocaine abuse. NeuroImage 2008; 42: 869-78.

[3] Descamps M, Hyare H, Stebbing J, Winston A. Magnetic resonance imaging and spectroscopy of the brain in HIV disease. J HIV Ther 2008; $13: 55-8$.

[4] Georgiou MF, Gonenc A, Waldrop-Valverde D, et al. Analysis of the effects of injecting drug use and HIV-1 infection on 18F-FDG PET brain metabolism. J Nucl Med 2008; 49: 1999-2005.

[5] Suomela S, Cao L, Bowcock A, Saarialho-Kere U. Interferon alphainducible protein 27 (IFI27) is upregulated in psoriatic skin and certain epithelial cancers. J Invest Dermatol 2004; 122: 717-21.

[6] Wang L, Sun Y, Jiang M, Zheng X. Integrative decomposition procedure and Kappa statistics for the distinguished single molecular network construction and analysis. J Biomed Biotechnol 2009; 2009: 726-728.

[7] Storey JD. A direct approach to false discovery rates. J R Stat Soc, Ser B 2002; 64: 479-98.

[8] Wang Y, Joshi T, Zhang XS, Xu D, Chen L. Inferring gene regulatory networks from multiple microarray datasets. Bioinformatics (Oxford, England) 2006; 22: 2413-20.

[9] Huang da W, Sherman BT, Tan Q, et al. The DAVID Gene Functional Classification Tool: a novel biological module-centric algorithm to functionally analyze large gene lists. Genome Biol 2007; 8: R183.

[10] Dennis G, Jr., Sherman BT, Hosack DA, et al. DAVID: Database for Annotation, Visualization, and Integrated Discovery. Genome Biol 2003; 4: P3.

[11] Sun Y, Wang L, Jiang M, Huang J, Liu Z, Wolfl S. Secreted Phosphoprotein 1 Upstream Invasive Network Construction and Analysis of Lung Adenocarcinoma Compared with Human Normal Adjacent Tissues by Integrative Biocomputation. Cell Biochem Biophys 2009; 56: 59-71.

[12] Sun Y, Wang L, Lui L. Integrative decomposition procedure and Kappa statistics set up ATF2 ion binding module in Malignant Pleural Mesothelioma (MPM). Frontiers Electr Electron Eng China 2008; 3: 381-7.

[13] Wang L, Huang J, Jiang M. RRM2 Computational Phosphoprotein Network Construction and Analysis between No-Tumor Hepatitis/Cirrhotic Liver Tissues and Human Hepatocellular Carcinoma (HCC). Cell Physiol Biochem 2010; 26: 303-10.
[14] Wang L, Huang J, Jiang M, Zheng X. AFP computational secreted network construction and analysis between human hepatocellular carcinoma (HCC) and no-tumor hepatitis/cirrhotic liver tissues. Tumor Biol 2010. [Epub ahead of print].

[15] Wang L, Sun Y, Jiang M, Zhang S, Wolfl S. FOS proliferating network construction in early colorectal cancer (CRC) based on integrative significant function cluster and inferring analysis. Cancer Invest 2009; 27: 816-24.

[16] Devers M, Rouard N, Martin-Laurent F. Genetic rearrangement of the atzAB atrazine-degrading gene cassette from $\mathrm{pADP} 1:: \operatorname{Tn} 5$ to the chromosome of Variovorax sp. MD1 and MD2. Gene 2007; 392: 1-6.

[17] Erridge C, Kennedy S, Spickett CM, Webb DJ. Oxidized phospholipid inhibition of toll-like receptor (TLR) signaling is restricted to TLR2 and TLR4: roles for CD14, LPS-binding protein, and MD2 as targets for specificity of inhibition. J Biol Chem 2008; 283: 2474859.

[18] Lauer S, Kunde YA, Apodaca TA, Goldstein B, Hong-Geller E. Soluble MD2 increases TLR4 levels on the epithelial cell surface. Cell immunol 2009; 255(1-2): 8-16.

[19] Schnabl B, Brandl K, Fink M, et al. A TLR4/MD2 fusion protein inhibits LPS-induced pro-inflammatory signaling in hepatic stellate cells. Biochem Biophys Res Commun 2008; 375: 210-4.

[20] Scott MJ, Billiar TR. Beta2-integrin-induced p38 MAPK activation is a key mediator in the CD14/TLR4/MD2-dependent uptake of lipopolysaccharide by hepatocytes. J Biol Chem 2008; 283: 2943346.

[21] Hozumi Y, Fukaya M, Adachi N, et al. Diacylglycerol kinase beta accumulates on the perisynaptic site of medium spiny neurons in the striatum. Eur J Neurosci 2008; 28: 2409-22.

[22] Hozumi Y, Watanabe M, Otani K, Goto K. Diacylglycerol kinase beta promotes dendritic outgrowth and spine maturation in developing hippocampal neurons. BMC Neurosci 2009; 10: 99.

[23] Nakano T, Hozumi Y, Ali H, et al. Diacylglycerol kinase zeta is involved in the process of cerebral infarction. Eur J Neurosci 2006; 23: 1427-35.

[24] Nakano T, Iseki K, Hozumi Y, Kawamae K, Wakabayashi I, Goto K. Brain trauma induces expression of diacylglycerol kinase zeta in microglia. Neurosci Lett 2009; 461:110-5.

[25] Braun DC, Cao Y, Wang S, Garfield SH, Hur GM, Blumberg PM. Role of phorbol ester localization in determining protein kinase $\mathrm{C}$ or RasGRP3 translocation: real-time analysis using fluorescent ligands and proteins. Mol Cancer Ther 2005; 4: 141-50.

[26] Coughlin JJ, Stang SL, Dower NA, Stone JC. RasGRP1 and RasGRP3 regulate B cell proliferation by facilitating B cell receptorRas signaling. J Immunol 2005; 175: 7179-84. 
[27] Okamura SM, Oki-Idouchi CE, Lorenzo PS. The exchange factor and diacylglycerol receptor RasGRP3 interacts with dynein light chain 1 through its C-terminal domain. J Biol Chem 2006; 281: 36132-9.

[28] Ozaki N, Miura Y, Yamada T, Kato Y, Oiso Y. RasGRP3 mediates phorbol ester-induced, protein kinase $\mathrm{C}$-independent exocytosis. Biochem Biophys Res Commun 2005; 329: 765-71.

[29] Zheng Y, Liu H, Coughlin J, Zheng J, Li L, Stone JC. Phosphorylation of RasGRP3 on threonine 133 provides a mechanistic link between PKC and Ras signaling systems in B cells. Blood 2005; 105: 3648-54

[30] Lankat-Buttgereit B, Muller S, Schmidt H, Parhofer KG, Gress TM, Goke R. Knockdown of Pdcd4 results in induction of proprotein convertase $1 / 3$ and potent secretion of chromogranin $A$ and secretogranin II in a neuroendocrine cell line. Biology of the cell / under the auspices of the European Cell Biology Organization 2008; 100: 703-15.

[31] Schmid T, Jansen AP, Baker AR, Hegamyer G, Hagan JP, Colburn NH. Translation inhibitor Pdcd4 is targeted for degradation during tumor promotion. Cancer Res 2008; 68: 1254-60.

[32] Suzuki C, Garces RG, Edmonds KA, et al. PDCD4 inhibits translation initiation by binding to eIF4A using both its MA3 domains. Proc Natl Acad Sci USA 2008; 105:3274-9.

[33] Talotta F, Cimmino A, Matarazzo MR, et al. An autoregulatory loop mediated by miR-21 and PDCD4 controls the AP-1 activity in RAS transformation. Oncogene 2010. [Epub ahead of print].

[34] Wang Q, Sun Z, Yang HS. Downregulation of tumor suppressor Pdcd4 promotes invasion and activates both beta-catenin/Tcf and AP1-dependent transcription in colon carcinoma cells. Oncogene 2008; 27: $1527-35$.

[35] Wang X, Wei Z, Gao F, et al. Expression and prognostic significance of PDCD4 in human epithelial ovarian carcinoma. Anticancer Res 2008; 28: 2991-6.

[36] Shim JH, Choi HS, Pugliese A, et al. (-)-Epigallocatechin gallate regulates $\mathrm{CD} 3$-mediated $\mathrm{T}$ cell receptor signaling in leukemia through the inhibition of ZAP-70 kinase. J Biol Chem 2008; 283 : 28370-9.

[37] Smolej L, Vroblova V, Novosad J. Expression of ZAP-70 in patients with chronic lymphocytic leukemia may change significantly during the course of the disease. Int J Lab Hematol 2008; 30: 259-60; author reply 60 .

[38] Tabaczewski P, Nadesan S, Lim SH. Zap-70 positive chronic lymphocytic leukemia co-existing with Jak $2 \mathrm{~V} 671 \mathrm{~F}$ positive essential thrombocythemia: A common defective stem cell? Leukemia Res 2009; 33(6): 854-5.

[39] Wandroo F, Bell A, Darbyshire P, et al. ZAP-70 is highly expressed in most cases of childhood pre-B cell acute lymphoblastic leukemia. Int J Lab Hematol 2008; 30: 149-57.

[40] Zhu Y, Gao G. ZAP-mediated mRNA degradation. RNA Biol 2008; 5: 65-7.

[41] Holm R, Knopp S, Kumar R, et al. Expression of ZNF652, a novel zinc finger protein, in vulvar carcinomas and its relation to prognosis. J Clin Pathol 2008; 61: 59-63.

[42] Kumar R, Cheney KM, McKirdy R, et al. CBFA2T3-ZNF652 corepressor complex regulates transcription of the E-box gene HEB. J Biol Chem 2008; 283: 19026-38.

[43] Kumar R, Manning J, Spendlove HE, et al. ZNF652, a novel zinc finger protein, interacts with the putative breast tumor suppressor CBFA2T3 to repress transcription. Mol Cancer Res 2006; 4: 655-65.

[44] Aranda P, Valdivielso P, Pisciotta L, et al. Therapeutic management of a new case of LCAT deficiency with a multifactorial long-term approach based on high doses of angiotensin II receptor blockers (ARBs). Clin Nephrol 2008; 69: 213-8.

[45] Calabresi L, Favari E, Moleri E, et al. Functional LCAT is not required for macrophage cholesterol efflux to human serum. Atherosclerosis 2009; 204(1): 141-6.
[46] Nakhjavani M, Esteghamati A, Esfahanian F, Ghanei A, Rashidi A, Hashemi S. HbA1c negatively correlates with LCAT activity in type 2 diabetes. Diabetes Res Clin Pract 2008; 81: 38-41.

[47] Scarpioni R, Paties C, Bergonzi G. Dramatic atherosclerotic vascular burden in a patient with familial lecithin-cholesterol acyltransferase (LCAT) deficiency. Nephrol Dial Transplant 2008; 23: 1074; author reply -5 .

[48] Weber CL, Frohlich J, Wang J, Hegele RA, Chan-Yan C. Stability of lipids on peritoneal dialysis in a patient with familial LCAT deficiency. Nephrol Dial Transplant 2007; 22: 2084-8.

[49] Beyens G, Daroszewska A, de Freitas F, et al. Identification of sexspecific associations between polymorphisms of the osteoprotegerin gene, TNFRSF11B, and Paget's disease of bone. J Bone Miner Res 2007; 22: 1062-71.

[50] Chong B, Hegde M, Fawkner M, et al. Idiopathic hyperphosphatasia and TNFRSF11B mutations: relationships between phenotype and genotype. J Bone Miner Res 2003; 18: 2095-104.

[51] Cundy T, Hegde M, Naot D, et al. A mutation in the gene TNFRSF11B encoding osteoprotegerin causes an idiopathic hyperphosphatasia phenotype. Hum Mol Genet 2002; 11: 2119-27.

[52] Janssens K, de Vernejoul MC, de Freitas F, Vanhoenacker F, Van Hul W. An intermediate form of juvenile Paget's disease caused by a truncating TNFRSF11B mutation. Bone 2005; 36: 542-8.

[53] Vidal C, Brincat M, Xuereb Anastasi A. TNFRSF11B gene variants and bone mineral density in postmenopausal women in Malta. Maturitas 2006; 53: 386-95.

[54] Whyte MP, Singhellakis PN, Petersen MB, Davies M, Totty WG, Mumm S. Juvenile Paget's disease: the second reported, oldest patient is homozygous for the TNFRSF11B "Balkan" mutation (966_969delTGACinsCTT), which elevates circulating immunoreactive osteoprotegerin levels. J Bone Miner Res 2007; 22: 938-46.

[55] Moore EC, Barber J, Tripp RA. Respiratory syncytial virus (RSV) attachment and nonstructural proteins modify the type I interferon response associated with suppressor of cytokine signaling (SOCS) proteins and IFN-stimulated gene-15 (ISG15). Virol J 2008; 5: 116.

[56] Okumura A, Pitha PM, Harty RN. ISG15 inhibits Ebola VP40 VLP budding in an L-domain-dependent manner by blocking Nedd4 ligase activity. Proc Natl Acad Sci USA 2008; 105: 3974-9.

[57] Okumura F, Lenschow DJ, Zhang DE. Nitrosylation of ISG15 prevents the disulfide bond-mediated dimerization of ISG15 and contributes to effective ISGylation. J Biol Chem 2008; 283: 24484-8.

[58] Pattyn E, Verhee A, Uyttendaele I, et al. HyperISGylation of Old World monkey ISG15 in human cells. PLoS One 2008; 3: e2427.

[59] Vargas-Inchaustegui DA, Xin L, Soong L. Leishmania braziliensis infection induces dendritic cell activation, ISG15 transcription, and the generation of protective immune responses. J Immunol 2008; 180: 7537-45.

[60] Francis PJ, Hamon SC, Ott J, Weleber RG, Klein ML. Polymorphisms in $\mathrm{C} 2, \mathrm{CFB}$ and $\mathrm{C} 3$ are associated with progression to Advanced Age-Related Macular Degeneration associated with visual loss. J Med Genet 2009; 46(5): 300-7.

[61] Grochowalski A, Konieczynski J. PCDDs/PCDFs, dl-PCBs and HCB in the flue gas from coal fired CFB boilers. Chemosphere 2008; 73: 97-103.

[62] Jakobsdottir J, Conley YP, Weeks DE, Ferrell RE, Gorin MB. C2 and $\mathrm{CFB}$ genes in age-related maculopathy and joint action with $\mathrm{CFH}$ and LOC387715 genes. PLoS One 2008; 3: e2199.

[63] Lu QG, Li ZW, Na YJ, Ba SL, Sun YK, He J. Low SO2 emission from CFB co-firing MSW and bituminous. J Environ Sci (China) 2004; 16: 821-4.

[64] Ohkuma M, Noda S, Hongoh Y, Kudo T. Diverse bacteria related to the bacteroides subgroup of the CFB phylum within the gut symbiotic communities of various termites. Biosci Biotechnol Biochem 2002; 66: 78-84. 